Original Article

\title{
A COMPUTATIONAL APPROACH ON UNDERSTANDING STRUCTURAL INTERACTIONS OF ENVELOPE PROTEIN OF DENGUE VIRUS BOUND WITH SQUALENE, A PROTOTYPE ANTI-VIRAL COMPOUND
}

\author{
RAJKUBERAN CHANDRASEKARAN ${ }^{1}$, THIRUNAVUKKARASU SIVARAMAN ${ }^{*}$, SIVAPERUMAL \\ SIVARAMAKRISHNAN ${ }^{2}$, JAYASREE JS KUMAR ${ }^{3}$
}

1Department of Biotechnology, Karpagam Academy of Higher Education (Deemed to be University), Coimbatore 641021, Tamil Nadu, India, ${ }^{2}$ Department of Biotechnology, Bharathidasan University, Tiruchirappalli 620024, Tamil Nadu, India, ${ }^{3}$ Department of Molecular Biology, School of Biological Sciences, Madurai Kamaraj University, Madurai 625021, Tamil Nadu, India Email: eazhilarasen@yahoo.com

Received: 12 Sep 2018 Revised and Accepted: 04 Dec 2018

\section{ABSTRACT}

Objective: The objective of the work was to validate the structural binding affinity of Squalene with the envelope protein of Dengue virus by means of molecular simulations.

Methods: Three-dimensional (3D) structure of dengue 2 virus envelope protein was retrieved from Protein Data Bank PDB and Squalene compound from the ZINC database. Molecular docking between the E protein and Squalene were carried out by means of Auto Dock 4.2.

Results: Based on the study, it was observed that the binding/docking energy for the complex structure was calculated to be-5.55 kcal/mol. Critical residues to interact with $\mathrm{E}$ protein were scrutinized by analyzing the interface of the complex within $4 \AA$ A proximity. Residues such as Thr 48 , Glu 49 , Ala 50, Val 130, Leu 135, Ser 186, Pro 187, Thr 189, Gly 190, Leu 191, Phe 193, Leu 198, Leu 207, Thr 268, Phe 279, Thr 280, Gly 281, His 282 and Leu 283 were found to be non-covalently located around the squalene.

Conclusion: Scopes to design de novo anti-viral compounds to the dengue viruses by using squalene as a new class of template structure have also been concisely brought into fore.

Keywords: Anti-viral compounds, Dengue Virus, E protein, Molecular Dockings, Squalene

(C) 2019 The Authors. Published by Innovare Academic Sciences Pvt Ltd. This is an open access article under the CC BY license (http://creativecommons.org/licenses/by/4.0/) DOI: http://dx.doi.org/10.22159/ijpps.2019v11i1.29714

\section{INTRODUCTION}

In general, diseases can be defined as disorders of physiological functions in human beings. The diseases can be broadly classified into infectious and non-infectious diseases: infectious diseases are caused by pathogenic microorganisms like bacteria, viruses, fungi and the diseases can be spread from one person to another directly or indirectly; non-infectious diseases (such as cancer, diabetes and etc.) are generally caused by genetics, molecular dysfunctions, environmental factors or nutritional abnormalities [1]. Of the various infectious diseases, neglected tropical diseases (NTDs) comprise a group of diseases such as Buruli ulcer, Lymphatic filariasis, Chagas disease, Onchocerciasis, Dengue fever, Schistosomiasis, Dracunculiasis (guinea-worm disease) Soiltransmitted helminthiasis (ascariasis), Human African trypanosomiasis, Hookworm infections, Trichuriasis, Leishmaniasis, Trachoma, Leprosy and Zoonotic diseases and the NTDs are prevalent in developed and as well as developing countries according to the World Health Organization (WHO) [2]. A recent survey suggests that more than 1 billion people worldwide are affected by the NTDs and the people suffer from life-long physical pain and social stigmatization. Etiology of the NTDs is chiefly attributed to the unhygienic lifestyle and substandard sanitation practices [3].

Dengue is one of the NTDs and the disease is originated from the dengue viruses belong to Flavivirus, which is the genus of the family Flaviviridae. The dengue viruses cause Encephalitis in a human being and the people with encephalitis suffer from fever, seizures, severe headache, photophobia, cough and drowsiness [4]. There are no specific anti-viral drugs and vaccination to treat the dengue disease to date, though drugs such as ribavirin, acyclovir and EICAR are being used to treat the infections at the present juncture [5]. The dengue viruses are transmitted to human beings by arthropods such as Aedes aegypti and Aedes albopictus and outbreak of the diseases is severe especially in monsoon season of tropical countries exercising substandard sanitation [6].

The dengue virus consists of a single-stranded RNA encoding three structural proteins (capsid protein (C), pre-membrane protein (prM), and envelope protein (E)) and seven non-structural proteins (NS1, NS2A, NS2B, NS3, NS4A, NS4B, and NS5) [7]. The viral RNA is encapsulated by the structural proteins $\mathrm{C}$ and $\mathrm{E}$ and the protein $\mathrm{E}$ play essential roles in fusing the viral membrane with host membrane under acidic environments [8]. It has been substantiated that hydrophobic residues of beta-hairpin of the protein $E$ comprising of residues from 268 to 280 are crucial for the protein E conformational changes leading to the fusion processes [9]. In this background, a few studies reported in the literature have shown that small chemical molecules that are targeting to interact with the hydrophobic residues present in the groove of the envelope protein E would pave a way to develop anti-viral chemical compounds against the dengue viruses.

In the present study, we have examined specific structural interactions between the E protein and triterpenoid squalene, which has been recently proven to exhibit larvicidal activity against Aedes aegypti [10]. Outputs of the study demonstrated that the squalene is a promising prototype for developing a novel class of antiviral compounds against the dengue viruses.

\section{MATERIALS AND METHODS}

Structural analyses and molecular docking

Three-dimensional (3D) structure of dengue 2 virus envelope protein bound with n-octyl-beta-D glucoside was retrieved from Protein Data Bank (PDB, https://www. rcsb. org/) and PDB ID of the protein is 10KE, which was dimer in the crystal state. The 
monomeric form of the protein consisting of 394 standard amino acids (ranging from 281 to 674 ) and also bereft of the ligand and water molecules were prepared, energy minimized and used for the molecular docking studies of the present studies. The 3D structure of the small chemical molecule, squalene $\left(\mathrm{C}_{30} \mathrm{H}_{50}\right.$, ZINC 06845904), was retrieved in mol2 structural format from the ZINC database [11].

Molecular dockings between the envelope protein and the squalene were carried out by means of Auto Dock 4.2 [12] wherein the protein and the ligand was kept as rigid and flexible, respectively. The docking parameters such as genetic runs, populations, energy evaluations, generations, the rate of gene mutation and rate of crossover were set as $100,500,25,000,000,27,000,0.02$ and 0.8 , respectively. Grid dimensions of 84 x 88 x $92 \AA$ were used to cover the entire cavity (which were present on core region of the protein) of the envelope protein and as well the n-octyl-beta-D-glucoside binding groove of the protein.

After successful completion of the docking processes, the docked conformations of the ligands were clustered with a tolerance limit of $1 \AA \AA$. Based on the cluster patterns and docking energies, a representative protein-ligand complex was generated and the complex structure was further used in order to scrutinize various non-bonding network interactions between the molecules. The structural interactions between the envelope protein and the squalene of the docking complex structure were viewed, analyzed and represented using molecular visualization tool PyMol (http://www.pymol.org).

\section{RESULTS AND DISCUSSION}

Infections due to dengue diseases are a serious medical problem in most tropical countries. To date, there are no specific medications to mitigate or to eradicate the diseases. There are reports in the literature demonstrating quests to identify lead molecules that are capable of retarding the functions of either structural or nonstructural proteins of the dengue viruses [13]. In this context, it is worthy of mentioning that a few small molecular inhibitors to the non-structural proteins of the dengue viruses have been reported as probed by a variety of computational strategies. For instances, Sivakumar et al. [14] designed a few novel ribavirin triphosphate analogs as antagonists to NS5-methyltransferase of dengue viruses by means of an array of in silico strategies and the lead compounds may eventually be developed as anti-viral drugs against the dengue viruses. Moreover, the research studies also rationalized that the novel ribavirin analogs presumably may not cause hemolytic anemia to the patients supposed to administrate them as the compounds showed weak affinities towards the JAK2 enzyme playing essential roles in erythropoiesis. Qamar et al. [15] postulate through a computational approach that flavanoids (Deoxycalyxin A, 3,5,7,3', $4^{\prime}-$ pentahydroxyflavonol-3-0-beta-D-galactopyranoside, (3R)-3',8Dihydroxyvestitol, Sanggenon 0, Epigallocatechin gallate and Chamaejasmine) inhibited the NS1 (by blocking through the Asparagin 130 glycosylation site), an important regulatory element in the replication processes of dengue viruses. Ariza et al. [16] showed that molecular dockings between curcumin and RdRp domain of the NS5 protein, also a key element in the viral replication, with a binding energy of $-6.2 \mathrm{Kcal} / \mathrm{mol}$. Rufaidah Othman et al. [17] demonstrated the binding interactions of antibiotics pinostrobin (with binding energy of $-5.33 \mathrm{Kcal} / \mathrm{mol}$ ), doxycycline (with binding energy of-5.15 Kcal/mol), 4-hydroxypanduratin A (with binding energy of $-4.45 \mathrm{Kcal} / \mathrm{mol}$ ), meclofenamic acid (with binding energy of-3.64 Kcal/mol) androlitetracycline (with binding energy of-3.21 Kcal $/ \mathrm{mol}$ ) against NS2B-NS3 proteases of dengue viruses. Nasution Aini and Tambunan [18] listed out 18 promising leads to chemotherapy treatments of the dengue diseases based on the comprehensive analyses of molecular docking complexes of 308 diverse molecules bound with NS2B-NS3 proteases of the dengue viruses.

E protein of dengue virus is a key regulatory element in the fusion process with the host cells. The fusion process involves host entry and attachment; internalization of the viral genome in the host is accomplished by receptor-mediated, clathrin-dependent endocytosis [19]. Hence, the non-structural proteins that are playing essential roles in integrating virus genome with the host organisms would also act as druggable targets. In other words, preventing interactions between the surface proteins of dengue virus with membrane proteins of host cells is also an attracting strategy in the chemotherapy treatments of dengue diseases. There are a few limited studies that have been conducted on the molecular interactions between $\mathrm{E}$ protein and various compounds derived from plant sources by using experimental and as well as computational approaches. For instance, Asif Mir et al. [20] performed molecular simulation studies on E protein with a few flavonoids (baicalein, fisetin, naringenin, quercetin) and showed that flavonoids could possible interrupt the fusion process by inhibiting the hydrophobic residues present in the groove of the envelope protein E. The binding affinities of the ligands baicalein, naringenin, fisetinquercetin were reported to be- $8.5 \mathrm{Kcal} / \mathrm{mol},-8.1 \mathrm{kcal} / \mathrm{mol},-7.3$ $\mathrm{kcal} / \mathrm{mol}$ and $-7.1 \mathrm{kcal} / \mathrm{mol}$, respectively, suggesting the structural interactions between the E protein and the flavonoids are highly specific in nature.

As discussed above and as well numerous reports in the literature, primary validation of leads/drugs through in silico approach against various infectious diseases is popularizing among research fraternity due to the feasibility of the drug discovery process [21]. Currently, experiments pertaining to a high-throughput virtual screening of phytochemicals against target sites/molecules of infectious agents have a profound significance in the development of lead compounds [22]. In the present study, we have examined specific structural interactions between the triterpenoid squalene and protein $\mathrm{E}$ of the dengue virus by means of molecular docking methods. The squalene is a triterpenoid compound found in latex and resins of plants; functionally the squalene bears defense mechanism against pathogens. As reported in the literature, squalene has a therapeutic regimen such as antimicrobial, anticancer, antioxidant, chemopreventive, gastropreventive and hepatoprotective, pesticide, antitumor and sunscreen properties [23].

In the recent reports of the research group [10], the effects of latex extract from $E$. antiquorum on the various stages of instar larvae of A. aegypti and C. quinquefasciatus have been examined by using systematic experimental methods. Of the different solvent extracts of latex, the chloroform extract depicted remarkable growth inhibitory activity against different instar larvae of $A$. aegypti and $C$. quinquefasciatus vis-à-vis to other solvent extracts of latex used in the studies. The $\mathrm{LC}_{50}$ values of the chloroform extract against the larval stages of II, III and IV of A. aegypti were found to be 68.7, 82.2 and 90.5 (ppm) respectively; similarly, The $\mathrm{LC}_{50}$ values of the chloroform extract against the larval stages of II, III and IV of $C$. quinquefasciatus were found to be 70.0, 72.24 and 68.9 (ppm), respectively. The chloroform extract was then subjected to GC-MS analysis in order to understand the phytochemical composition of the extract and the data suggested that squalene is the abundant phytochemical accounting about $18 \%(\mathrm{w} / \mathrm{w})$ of the dry extract. Taken together, the anti-mosquitocidal activity of the latex chloroform extract of E. antiquorum could be attributed to the presence of squalene in the extract. However, the mechanism by which the squalene function as the anti-mosquitocidal activity is left unaddressed to date to the best of our knowledge.

The physicochemical properties of the squalene and the active site of the dengue virus envelope protein was found to be compatible with each other and the data provided the cue for studying interactions between the two molecules. The active site of the envelope protein is highly hydrophobic in nature and the protein is essential to fuse the viruses with host systems. Interestingly, the squalene depicted structural and functional complementary features to be accommodated in the active site cavity of the envelope protein. The overall three-dimensional conformations of the squalene and envelop protein are depicted in fig. 1 in sticks and surface models, respectively. The molecular interactions between the squalene and the envelope protein were examined using AutoDock 4.2 docking tool as described in the method section. Overall binding mode between the squalene and envelop protein, as simulated from the molecular docking tool, is illustrated in the fig. 2 and the binding/docking energy for the complex structure was calculated to be-5.55 kcal/mol. Crucial residues of the envelope protein to interact with the squalene were scrutinized by analyzing the 
interface of the complex within $4 \AA$ proximity. Residues such as Thr 48, Glu49, Ala 50, Val 130, Leu 135, Ser 186, Pro 187, Thr 189, Gly 190, Leu 191, Phe 193, Leu 198, Leu 207, Thr 268, Phe 279, Thr 280, Gly 281, His 282 and Leu 283 were found to be non-covalently located around the squalene. The comprehensive analyses of the complex at overall molecular and as well at residue level suggested the squalene could be fully accommodated in the hydrophobic cavity located in the central part of the envelope protein. Meanwhile, it would be favorable to confirm the in silico rationalization of the specificities brought out into fore herein for the binding interactions between the squalene and the envelope protein at residue level resolution through experimental evidence in near future.

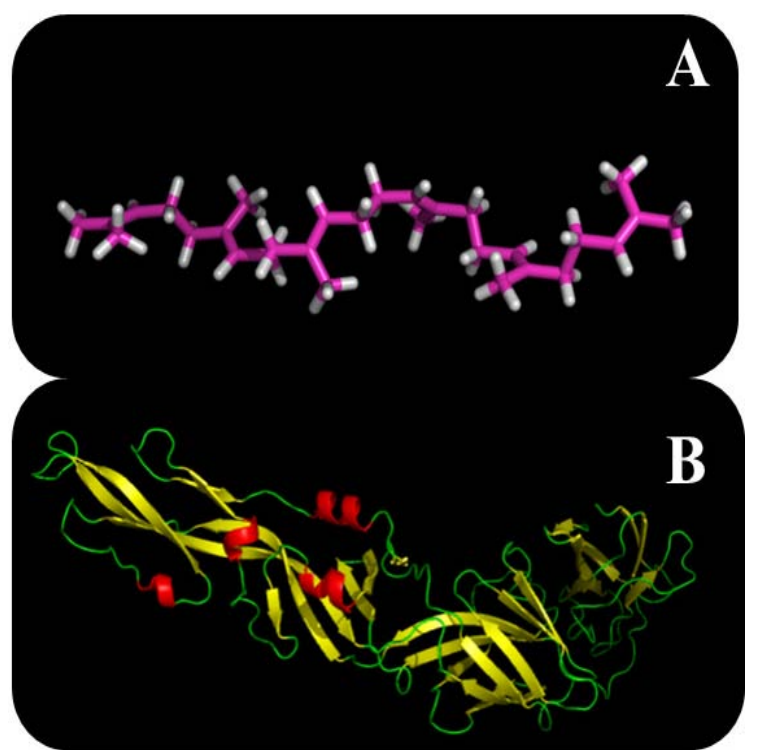

Fig. 1: The three-dimensional structures of A) Squaleen and B) Envelop protein of dengue virus are depicted in sticks and surface models, respectively

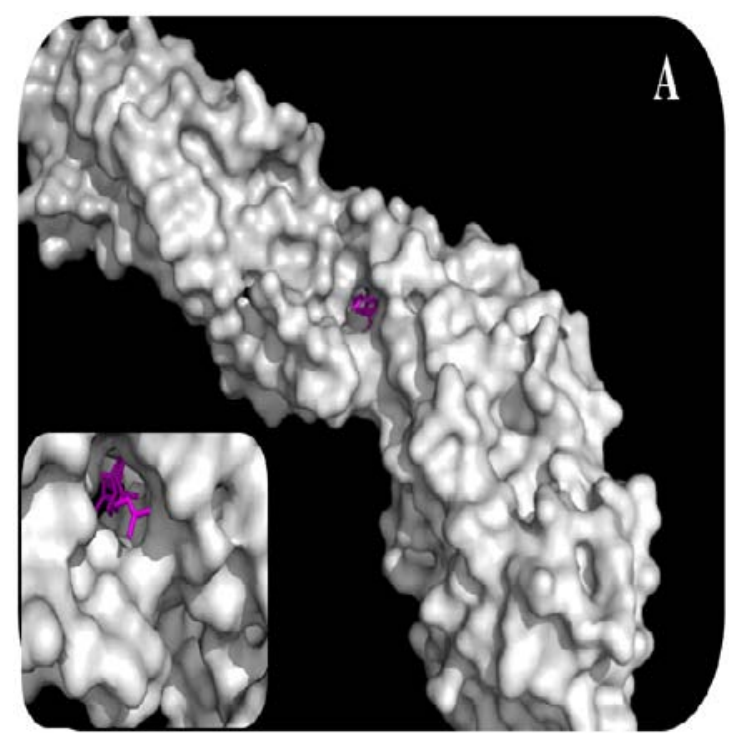

Fig. 2: A) Docked conformation of the squalene and envelop protein is depicted in magenta sticks and gray surface models, respectively. The inset fig. shows zoomed part of the squalene and hydrophobic pocket of the envelop protein

Fig. 2B: Snapshot representing residues of envelop protein present in the $4 \AA$ A proximity of squalene (stick model in magenta). The Residues such as Thr 48, Glu49, Ala 50, Val 130, Leu 135, Ser 186, Pro 187, Thr 189, Gly 190, Leu 191, Phe 193, Leu 198, Leu 207, Thr 268, Phe 279, Thr 280, Gly 281, His 282 and Leu 283 are shown in sticks models (hydrogen atoms are excluded for sake of clarity) with carbon, nitrogen and oxygen atoms painted as gray, blue and red, respectively.

\section{CONCLUSION}

In conclusion, the computational analyzes revealed that the squalene is capable of strongly to interact on the hydrophobic cavity of the dengue virus envelop protein. By binding to the hydrophobic pocket, the squalene would presumably inactivate the envelope protein playing essential roles in fusing dengue viruses with host organisms. Hence, the squalene can be used as a template structure to design highly efficient de novo anti-viral compounds against dengue viruses. To be brief, in the de novo drug designing, squalene as a seed molecule with the defined growing site(s) would be placed on the hydrophobic binding groove consisting of putative active site residues of the envelope protein and small molecular chemical compounds would then be generated by means of 'grow genetic algorithm'. Moreover, the algorithm can also be properly tuned in terms of fragment library, toxic library and as well other drug designing parameter as required to bring desired drug-likeness compounds against dengue viruses in near future.

\section{ACKNOWLEDGMENT}

The author TS would like to register his sincere thanks to the computational facility at KAHE for generously allowing to use various tools mentioned in the research work.

\section{AUTHORS CONTRIBUTIONS}

TS performed the computational part of the research work. TS and CRK drafted the manuscript. SRK, CRK and JSJ conceived the work.

\section{CONFLICTS OF INTERESTS}

Authors declare no conflicts of interest

\section{REFERENCES}

1. Kenneth M Boyd. Disease, illness, sickness, health, healing and wholeness: exploring some elusive concepts. J Med Ethics: Medical Humanities 2000;26:9-17.

2. World Health Organization. Neglected Tropical Diseases. Program; 2017. Available from: http://www.who.int/ neglected_diseases/en. [Last accessed on 10 Aug 2018]

3. Amal Mitra, Anthony Mawson. Neglected tropical diseases: epidemiology and global burden. Trop Med Infec Dis 2017;2:36.

4. Castilla, Luana Piccini, Elsa B Damonte. Dengue virus entry and trafficking: perspectives as antiviral target for prevention and therapy. Future Virol 2015;10:625-45.

5. Low, Eng Eong Ooi, Subhash G Vasudevan. Current status of dengue therapeutics research and development. J Infect Dis 2017;215 (Suppl 2):S96-S102.

6. Carrington LB, Simmons CP. Human to mosquito transmission of dengue viruses. Front Immunol 2014;5:290.

7. Rushika Perera, Richard J Kuhn. Structural proteomics of dengue virus. Curr Opin Microbiol 2008;11:369-77.

8. Laura A Byk, Andrea V Gamarnik. Properties and functions of the dengue virus capsid protein. Annu Rev Virol 2016;29:263-81.

9. Modis Y, Ogata S, Clements D, Harrison SC. A ligand-binding pocket in the dengue virus envelope glycoprotein. Proc Natl Acad Sci USA 2003;100:6986-91.

10. Rajkuberan C, Sathishkumar G, Prabukumar P, Muthukumar K, Sivaramakrishnan S. Intrinsic studies of Euphorbia antiquorum $L$. latex extracts against human bacterial pathogens and mosquito vector Aedes aegypti, Culex quinquefasciatus (Diptera: Culicidae). Biocatal Agric Biotechnol 2017;10:75-82.

11. Irwin, Sterling, Mysinger, Bolstad, Coleman. ZINC: a free tool to discover chemistry for biology. J Chem Inf Model 2012;52:1757-68.

12. Morris GM. Automated docking using a Lamarckian genetic algorithm and an empirical binding free energy function. J Comput Chem 1998;19:1639-62. 
13. Qian Xie, Bao Zhang, Jian Hai Yu, Qinghua Wu, Fangji Yang, Hong Cao, et al. Structure and function of the non-structural protein of dengue virus and its applications in antiviral therapy. Curr Top Med Chem 2017;17:3.

14. Sivakumar D, Sivaraman T. In silico designing and screening of lead compounds to NS5-methyltransferase of dengue viruses. Med Chem 2011;7:655-62.

15. Qamar, Arooj Mumtaz, Rabbia Naseem, Amna Ali, Tabeer Fatima, Tehreem Jabbar, et al. Molecular docking based screening of plant flavonoids as dengue NS1 inhibitors. Bioinformation 2014;10:460-5.

16. Ariza Leidy Lorena Garcia, Germán Alberto Tellez Ramirez. Molecular cloning, modelling and docking with curcumin of the dengue virus 2 NS5 polymerase domain. LF Castillo. eds. Advances in computational biology, advances in intelligent systems and computing 232. Springer International Publishing Switzerland; 2014.

17. Rufaidah Othman, Rozana Othman, Aida Baharuddin, Nagasundara Ramanan Ramakrishnan, Noorsaadah Abd Rahman, Rohana Yusof, et al. Molecular docking studies of selected medicinal drugs as dengue virus-2 protease inhibitors. Sains Malaysiana 2017;46:1865-75.
18. Nasution Aini, Tambunan. Virtual screening of commercial cyclic peptides as NS2B-NS3 protease inhibitor of dengue virus serotype 2 through molecular docking simulation. IOP Conf Series: Materials Science and Engineering; 2017. p. 188.

19. Joe Grove, Mark Marsh. The cell biology of receptor-mediated virus entry. J Cell Biol 2011;195:1071-82.

20. Asif Mir, Humaira Ismatullah, Sobiah Rauf, Umar Niazi. Identification of bioflavonoid as fusion inhibitor of dengue virus using molecular docking approach. Informatics Medicine Unlocked 2016;3:1-6.

21. Parikesit AA, Kinanty Tambunan US. Screening of commercial cyclic peptides as inhibitor envelope protein dengue virus (DENV) through molecular docking and molecular dynamics. Pak J Biol Sci 2013;16:1836.

22. Lin MH. Membrane undulation induced by NS4A of Dengue virus: a molecular dynamics simulation study. J Biomol Struct Dyn 2014;32:1552.

23. Duraisamy Gomathi, Manokaran Kalaiselvi, Ganesan Ravikumar, Kanakasabapathi Devaki, Chandrasekar Uma. GCMS analysis of bioactive compounds from the whole plant ethanolic extract of Evolvulusalsinoides (L.) L. J Food Sci Technol 2015:52;1212-7. 